\title{
COMPLICAÇÕES E INTERCORRÊNCIAS ASSOCIADAS AO EDEMA DE BRAÇO NOS TRÊS PRIMEIROS MESES PÓS MASTECTOMIA ${ }^{1}$
}

Marislei Sanches Panobianco ${ }^{2}$ Marli Villela Mamede ${ }^{3}$

Panobianco MS, Mamede MV. Complicações e intercorrências associadas ao edema de braço nos três primeiros meses pós mastectomia. Rev Latino-am Enfermagem 2002 julho-agosto; 10(4):544-51.

Este estudo buscou identificar complicações, intercorrências e aparecimento de edema pós cirurgia por câncer de mama, e fatores que poderiam estar predispondo ao linfedema do braço do lado operado. É um estudo descritivo, prospectivo e de abordagem quantitativa. A população constou de 17 mulheres submetidas à cirurgia unilateral por câncer de mama, em um hospital-escola. A coleta de dados deu-se por meio do acompanhamento semanal dos sujeitos, nos três meses pós-cirurgia. 0 edema apareceu em 11 mulheres, sendo, em nove, de grau leve, e, em duas de grau moderado. É importante lembrar que o edema leve poderá tornar-se grave se não tratado, devidamente. Observou-se, entre as mulheres com edema, complicações, intercorrências, e outras variáveis. Os resultados revelam a importância do acompanhamento de enfermagem no pós-operatório, e a necessidade de um melhor preparo dos profissionais para a orientação de pacientes, com o objetivo de prevenir o linfedema.

DESCRITORES: linfedema, mastectomia, câncer da mama

\section{COMPLICATIONS AND INTERCURRENCES ASSOCIATED WITH ARM EDEMA IN THE FIRST THREE MONTHS FOLLOWING MASTECTOMY}

This study aimed at identifying complications, intercurrences and the appearance of edema following breast cancer surgery as well as factors that could predispose to arm lymphedema on the operated side. It is a descriptive and prospective study based on a quantitative approach. The subjects consisted of 17 women submitted to unilateral mastectomy in a University Hospital. Data were collected by means of a weekly post-operative follow-up of each patient for a period of three months. Edema was detected in 11 women, nine of wich were in mild level and two in moderate level. It must be emphasized that even mild-level edema could become severe if not treated appropriately. Among the women with edema, complications, intercurrences and other variables were observed. The results revealed the importance of nursing follow-up during the mastectomy post-operative period as well as the need for better staff training for patient guidance aiming at edema prevention.

DESCRIPTORS: Iymphedema, mastectomy, breast cancer

\section{COMPLICACIONES E INTERCURRENCIAS ASOCIADAS AL EDEMA DE BRAZO EN LOS TRES PRIMEROS MESES DESPUÉS DE LA MASTECTOMÍA}

Este estudio buscó identificar complicaciones, intercurrencias y el aparecimiento del edema después de la cirugía por cáncer de mama y factores que pueden estar facilitando el aparecimiento del linfodema del brazo del lado operado. Es un estudio descriptivo, prospectivo y de abordaje cuantitativa. La población fue formada por 17 mujeres sometidas a cirugía unilateral por cáncer de mama en un hospital escuela. La recolección de datos ocurrió a través del seguimiento semanal de los sujetos, en los tres meses después de la cirugía. El edema apareció en 11 mujeres, siendo en nueve de grado leve y en dos de grado moderado. Es importante recordar que el edema leve podrá tornarse grave si no es tratado. Se observó entre las mujeres con edema, complicaciones, intercurrencias y otras variables. Los resultados revelan la importancia del seguimiento de enfermería en la fase posquirúrgica y la necesidad de una mejor preparación de los profesionales para la orientación de pacientes con el objetivo de prevenir el linfodema.

DESCRIPTORES: linfodema, mastectomía, cáncer de mama

\footnotetext{
${ }_{1}^{1}$ Dissertação de Mestrado apresentada ao Programa de Pós-Graduação da Área de Enfermagem em Saúde Pública - Subvenção do CNPq; ${ }^{2}$ Doutoranda do Departamento de Enfermagem Materno-Infantil e Saúde Pública, e-mail: marislei@eerp.usp.br; ${ }^{3}$ Professor Titular. Escola de Enfermagem de Ribeirão Preto, da Universidade de São Paulo, Centro Colaborador da OMS para o desenvolvimento da pesquisa em enfermagem
} 


\section{INTRODUÇÃO}

O linfedema é um incômodo físico e emocional para as mulheres mastectomizadas, quando observamos que muitas delas experimentam depressão, ansiedade, e chegam a necessitar de seguimento psicológico ou psiquiátrico.

O linfedema pós mastectomia causa para a paciente, não somente o dano estético, mas também o prejuízo funcional do membro afetado, e sérias conseqüências mentais, levando, ocasionalmente, a condições que ameaçam a vida. O linfedema do membro superior homolateral à cirurgia é uma das mais estressantes experiências para a paciente e pode preceder um linfangiossarcoma ${ }^{(1)}$.

O braço com linfedema vem a ser o foco de atenção de outras pessoas, e pode ocorrer, então, perda de interesse nas atividades sociais por parte da mulher que apresenta essa complicação. Atenta-se, também, para o fato de que com o aumento do linfedema, podem, também, aumentar as dificuldades na realização das tarefas em casa e no trabalho. Lembram ainda, que as mulheres com linfedema do braço pós mastectomia sentem-se aflitas quando se faz necessário mudar o estilo de roupa que usavam. Isso pode vir a causar perda de interesse com a aparência, e a perda da auto-estima contribui, então, para dificultar o relacionamento interpessoal e sexual ${ }^{(2)}$.

Além desses transtornos físicos e emocionais, constatamos, em nossa prática profissional, que o linfedema tem aparecido no período de até três meses após a cirurgia.

Diante de tais inquietações, assumimos o desafio de, num acompanhamento de três meses de pósoperatório de mulheres mastectomizadas, buscar identificar complicações e intercorrências que poderiam estar influenciando na etiologia do linfedema.

\section{LINFEDEMA E CÂNCER DE MAMA}

O linfedema é o edema de parte do corpo, que ocorre como resultado de uma insuficiência no sistema linfático ${ }^{(3)}$.

Vários autores somente consideram edema significante aquele cuja diferença de medidas, quando da realização da cirtometria* dos membros superiores for igual ou maior que $3 \mathrm{~cm}^{(4)}$.

Para outros, a simples diferença de 1 a $1,5 \mathrm{~cm}$ pode estabelecer um diagnóstico de linfedema, e a uma diferença inferior a $3 \mathrm{~cm}$, o linfedema é considerado leve; de 3 a $5 \mathrm{~cm}$, moderado; e o linfedema severo é uma diferença superior a $5 \mathrm{~cm}$, quando da comparação das medidas realizadas por meio da cirtometria de ambos os membros $^{(5)}$.

A literatura que trata dos fatores determinantes do linfedema pós cirurgia por câncer de mama, cita vários, dentre os quais podemos destacar: infecção; linfangite e celulite; radioterapia; obesidade; ceroma; nódulos linfáticos positivos; demora na cicatrização da ferida; dissecção ampliada de axila; curativo compressivo e imobilização do braço no pós-operatório.

No que se refere à severidade do linfedema pósmastectomia, os índices apresentam-se de forma variada entre os autores que têm estudado essa complicação. O valor estimado da incidência para linfedema moderado é de $25 \%$, e de $10 \%$ para linfedema severo ${ }^{(6)}$. Os valores da incidência do linfedema pós mastectomia chegam a variar de 3 a $80 \%{ }^{(4-7)}$

Fazendo um levantamento das 344 mulheres atendidas no Núcleo de Ensino, Pesquisa e Assistência na Reabilitação de Mastectomizadas - REMA, do Departamento de Enfermagem Materno-Infantil e Saúde Pública da Escola de Enfermagem de Ribeirão PretoUSP, num período de 6 anos, verificamos que 43 delas (16\%) já chegaram com linfedema de 3 a $4,5 \mathrm{~cm}$, e que $27(63 \%)$ dessas 43 pacientes não tinham ainda três meses de pós operatório.

Diante dessas considerações, procuramos, neste trabalho, fazer um acompanhamento, no período dos três primeiros meses de pós-operatório de mulheres submetidas à cirurgia por câncer de mama, na tentativa de identificar complicações e intercorrências que poderiam estar predispondo ao aparecimento de linfedema.

\section{OBJETIVOS}

Neste trabalho, temos, como objetivos, identificar:

\footnotetext{
* Cirtometria é a medida circular dos membros superiores com pontos de referência fixos, para cada cliente, com o intuito de verificar a existência e o grau de linfedema. (Beuttenmuller L. Mastectomia - fisioterapia em mastectomizadas, 1985)
} 
a presença de complicações/intercorrências pós cirurgia por câncer de mama; a partir do primeiro dia de pós operatório, a presença de níveis de edema de braço de mulheres com cirurgia por câncer de mama; associação entre o aparecimento de linfedema e complicações/ intercorrências.

\section{METODOLOGIA}

População em estudo

Fizeram parte de nosso estudo mulheres admitidas com diagnóstico de câncer de mama, unilateral, e indicação de cirurgia, em um hospital-escola.

A amostra representou $25 \%$ dos casos de mulheres submetidas à cirurgia de mama por câncer de mama (correspondente a 17 mulheres), no período de um ano, no referido hospital. Esse tamanho amostral foi fixado a partir do cálculo da média anual de cirurgias realizadas neste hospital, nos últimos cinco anos anteriores ao estudo. Para ser incluída na amostra, a paciente deveria ter sido submetida à cirurgia unilateral de mama, por câncer de mama; aceitar participar da pesquisa e da visita domiciliária, quando necessária.

Procedimentos metodológicos

Obtivemos aprovação, para o desenvolvimento da presente pesquisa, pelo Comitê de Ética do hospitalescola em que ela foi realizada. As mulheres que se enquadravam nos critérios estabelecidos para inclusão na amostra, foram convidadas a participar da pesquisa, após uma explanação de seus objetivos, no período préoperatório.

Após a aquiescência e documentação do interesse, para a coleta de dados, as mulheres foram acompanhadas pela pesquisadora, diariamente, na fase de internação; na alta hospitalar e, semanalmente, pósalta hospitalar, até o terceiro mês pós-cirurgia, sendo que esse acompanhamento foi feito no próprio hospital, no domicílio, ou ainda, na residência de amigos ou familiares em que as mulheres residentes em cidades distantes de Ribeirão Preto ficavam hospedadas.

A coleta de dados foi auxiliada por entrevistas e observações; foram tomados dados dos prontuários das pacientes e anotados em formulário específico.
No período de acompanhamento das pacientes, procurou-se identificar informações referentes a: incisão cirúrgica; curativo compressivo e imobilização do membro superior do lado operado; medicações prescritas e tratamentos complementares à cirurgia; drenos aspirativos; condições do membro superior homolateral à cirurgia; complicações e intercorrências. Em todas as visitas era verificado o peso e realizada a cirtometria.

Optamos por analisar todas as pacientes que apresentaram diferença igual ou maior a $1 \mathrm{~cm}$ entre os braços, em qualquer dos pontos mensurados ${ }^{(5)}$. Todos os dados foram anotados em formulário específico e submetidos à análise comparativa da freqüência absoluta e percentual entre as variáveis estudadas.

\section{RESULTADOS E DISCUSSÃO}

\section{Caracterização da amostra}

A amostra foi constituída por 17 mulheres submetidas à cirurgia de mama, por câncer, no período de abril a agosto de 1997, num hospital-escola da cidade de Ribeirão Preto. Ao caracterizar os sujeitos estudados, procuramos identificá-los de acordo com a idade, a ocupação, o grau de instrução, o estado civil, a procedência e a raça.

Pudemos verificar que a faixa etária variou de 38 a 72 anos, sendo que $11(64,7 \%)$ tinham mais de 50 anos. Identificamos que 15 mulheres $(88,2 \%)$ eram donas-de-casa, uma $(5,9 \%)$, faxineira, e uma $(5,9 \%)$, estudante. Treze(76,5\%) tinham primeiro grau incompleto, uma $(5,9 \%)$ tinha primeiro grau completo, duas $(11,8 \%)$ eram analfabetas, e uma (5,9\%) tinha curso superior. Doze mulheres $(70,6 \%)$ eram casadas, uma $(5,9 \%)$, solteira, três $(17,6 \%)$, viúvas, e uma $(5,9 \%)$, separada do marido. Em relação à procedência, a maioria, ou seja, 11 mulheres $(64,7 \%)$ residiam em Ribeirão Preto ou cidades da região.

Nossa amostra constou de 16 mulheres brancas $(94,1 \%)$ e uma da raça negra $(5,9 \%)$.

Ao identificar os aspectos relacionados ao câncer de mama, procuramos verificar a lateralidade da mama afetada, o estadiamento clínico da doença, o tipo de cirurgia, bem como outros tratamentos realizados e conhecimento sobre o cuidado com o braço.

Em relação à lateralidade da mama afetada, verificamos uma freqüência de $58,8 \%$ para a mama 
esquerda (dez pacientes), e de $41,2 \%$ para a mama direita (sete pacientes).

Em nosso estudo, utilizamos a classificação TNM (tumor, nódulo, metástase) do câncer de mama, da União Internacional Contra o Câncer (UICC) ${ }^{(8)}$, por estar de acordo com a que é utilizada pelo serviço onde foram coletados os dados. Das mulheres estudadas, uma $(5,9 \%)$ apresentava estadiamento clínico la duas mulheres $(11,8 \%)$ Ila, e três mulheres, Ilb. Em estadiamentos mais avançados, atingindo o nível III, identificamos quatro mulheres com o tumor em estágio Illa e cinco mulheres em estágio IIIb. Duas mulheres $(11,8 \%)$ apresentavam metástase à distância, enquadrando-se no estágio clínico IV.

Quanto ao tipo de cirurgia realizada, dez pacientes $(58,8 \%)$ foram submetidas à mastectomia radical modificada, sendo oito mastectomias à Patey $\mathrm{e}$ duas à Madden. Duas mulheres foram submetidas à quadrantectomia $(11,8 \%)$, e cinco $(29,4 \%)$ à nodulectomia, com esvaziamento axilar.

Dentre os tratamentos complementares à cirurgia, realizados pelos sujeitos estudados, estão a quimioterapia e a radioterapia. Foram submetidas à quimioterapia 14 mulheres $(82,3 \%)$. O tratamento quimioterápico resulta em significante aumento da ocorrência de edema de braço em mulheres submetidas à cirurgia por câncer de mama ${ }^{(9)}$.

A radioterapia foi realizada por 14 mulheres $(82,3 \%)$ que participaram deste estudo. Esse tratamento está relacionado a fatores predisponentes ao linfedema de braço pós mastectomia ${ }^{(10)}$.

Em relação ao que sabiam sobre os cuidados com o braço, as informações que as pacientes nos passavam não eram satisfatórias para a prevenção do linfedema. À medida que achávamos necessário, reforçávamos as orientações nesse sentido.

\section{Complicações e intercorrências pós cirurgia}

Ao fim do terceiro mês de pós-operatório, pudemos identificar que todas as mulheres apresentaram complicações e intercorrências, associadas ou não umas às outras. As complicações apresentadas pelas mulheres estudadas, revelando o surgimento de edema $(64,7 \%)$, foram: ceroma $(41,1 \%)$, deiscência $(47 \%)$, dor $(41,1 \%)$, limitação de amplitude de movimento $(64,7 \%)$, infecção $(23,5 \%)$, aderência $(41,1 \%)$, inflamação no local de inserção do dreno $(11,8 \%)$ e celulite $(5,9 \%)$.
Durante o período de acompanhamento, pudemos identificar algumas intercorrências com o membro superior homolateral à cirurgia, como: presença de secreção no local cirúrgico ou no local de inserção do dreno (54,5\%); outros problemas com o dreno aspirativo (27,3\%); picadas de inseto, contusões, cortes, punções venosas $(27,3 \%)$; flebite $(18,2 \%)$; hematoma $(18,2 \%)$; queimadura $(9,09 \%)$; micose de unha $(9,09 \%)$.

Identificamos, ainda, outros fatores que poderiam estar influenciando no aparecimento do linfedema, como: radioterapia e quimioterapia (82,3\%); não realização de exercícios com o braço do lado operado $(76,5 \%)$; estadiamento avançado da doença (III e IV - 64,7\%); cirurgia do lado não dominante (64,7\%); cirurgias com dissecção extensa (58,5\%); obesidade (52,9\%); aumento de atividade ocupacional, sem moderação $(27,3 \%)$.

Como o grande interesse deste trabalho foi a identificação de fatores que poderiam estar influenciando no aparecimento do edema de braço pós cirurgia por câncer de mama, procuramos identificar alguma correlação entre o edema e o surgimento de complicações, intercorrências e outros fatores.

Fatores relacionados ao edema do membro superior homolateral à cirurgia

O edema superior a $1 \mathrm{~cm}$ no membro do lado operado acometeu 11 mulheres $(64,7 \%)$ que fizeram parte da amostra. Ele apareceu em diferentes períodos, porém sempre coincidiu com outras complicações e/ou intercorrências (Quadro 1) ou foi precedido por elas.

Verificamos que, dentre as mulheres sujeitos deste estudo, nenhuma chegou a apresentar linfedema severo, ou seja, aquele cuja diferença entre as medidas dos braços é superior a $5 \mathrm{~cm}$. Das onze mulheres com linfedema, identificamos nove apresentando o de grau leve, cuja diferença entre as medidas dos braços é inferior a $3 \mathrm{~cm}$, e duas com linfedema de grau moderado, ou seja, com diferença entre 3 e $5 \mathrm{~cm}$.

Apesar desses resultados, com a maioria das mulheres apresentando linfedema de grau leve, consideramos importante a detecção precoce do seu aparecimento, uma vez, que quando não tratado, pode levar a sérias complicações.

O linfedema leve ou moderado pode vir a tornarse muito grave, chegando a ser um local de linfangiossarcoma subseqüente ${ }^{(11)}$. 
Quadro 1 - Evolução do edema do membro superior do lado operado, durante o período de acompanhamento de três meses

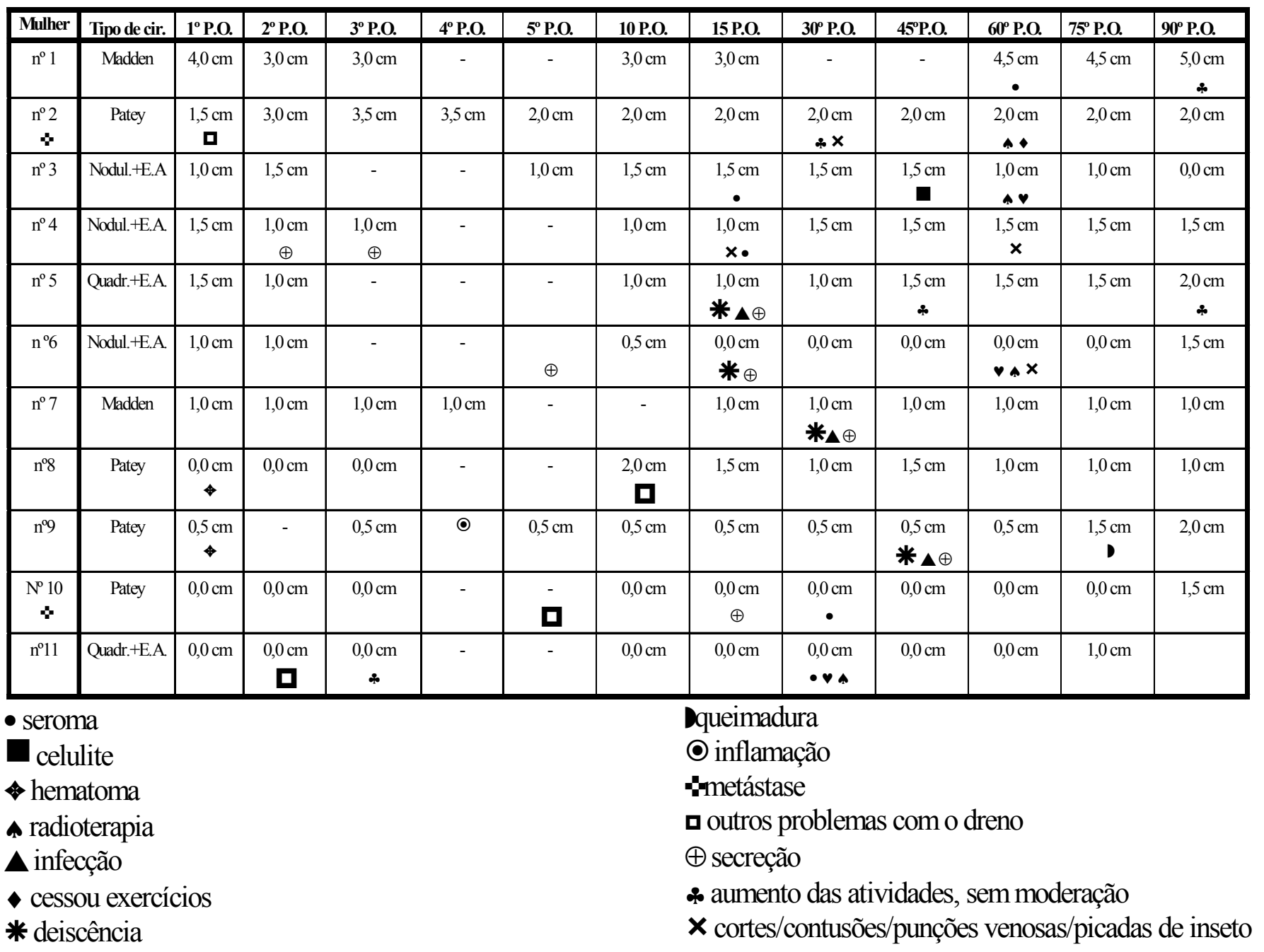

O edema de braço apareceu em cinco $(45,4 \%)$ mulheres que também apresentaram o ceroma como complicação pós-cirúrgica. Essas cinco mulheres representam $71,5 \%$ das que tiveram ceroma, complicação que pode contribuir para a formação de edema $^{(12)}$.

Ao relacionarmos o aparecimento de edema de membro superior com a presença de deiscência, observamos que quatro $(36,4 \%)$ das mulheres que apresentaram edema, tiveram deiscência de sutura da incisão cirúrgica. Essas quatro mulheres representam $50 \%$ das mulheres com deiscência.

O exercício terapêutico vem a ser um fator de importância no plano de assistência pós-operatória à paciente, para a prevenção do linfedema no membro superior homolateral e da limitação da mobilidade do ombro $^{(4-13)}$. Identificamos neste estudo, entre as 11 mulheres com edema, nove $(81,8 \%)$ apresentando limitação de amplitude do membro edemaciado, adicionando-se o fato de que dez $(90,9 \%)$ das mulheres com edema não faziam exercícios físicos regularmente.

Mulheres com câncer de mama notam limitado apoio ao exercício físico, por parte dos médicos e de pessoal de enfermagem. Alegam, ainda, que os profissionais de saúde não são bem informados no que diz respeito aos benefícios e exercícios adequados à mulher com câncer de mama ${ }^{(14)}$.

O estadiamento do tumor é decisivo na incidência de complicações pós-cirúrgicas ${ }^{(15)}$. Ao analisar a relação entre presença de edema e o estadiamento da doença, observamos que a maior freqüência de aparecimento de edema deu-se entre as pacientes com a doença nos estágios II, III e IV (dez - 90,9\%), apesar de ser esta uma característica da amostra como um todo.

O edema do membro superior do lado operado esteve presente em dez mulheres $(90,9 \%)$ que se 
submeteram ao tratamento por radioterapia e quimioterapia. Essas observações coincidem com as de vários autores que se reportam à quimioterapia e à radioterapia, como predisponentes ao aparecimento do edema do braço ${ }^{(9-10)}$. Convém ressaltar, no entanto, que $82,3 \%$ dos sujeitos estudados foram submetidos a esses tratamentos.

A incidência de linfedema é menor, quanto menos agressiva a cirurgia ${ }^{(16)}$. Em nosso estudo, foram submetidas à mastectomia radical modificada seis mulheres $(54,5 \%)$; foram, também, realizadas duas quadrantectomias $(18,2 \%)$ e três nodulectomias $(27,3 \%)$.

Seis $(54,5 \%)$ das mulheres com edema de braço apresentavam obesidade, que é citada por vários autores como fator que predispõe ao linfedema ${ }^{(10)}$.

Nosso estudo mostrou que oito $(72,8 \%)$ das mulheres que tiveram edema, foram submetidas à cirurgia no lado não dominante. Pensamos que, nesses casos, o aparecimento de edema estaria ligado ao fato de estarem começando mais tardiamente a movimentação do braço. Como o braço do lado dominante, que já é mais usado normalmente, está em melhores condições, a tendência é proteger o do lado operado, usando-o com menor freqüência. Outros estudos mostram uma pequena relação entre o aparecimento do edema e a dominância do membro homolateral à cirurgia ${ }^{(10)}$.

Associações com outras variáveis, citadas pelos autores como predisponentes ao aparecimento do edema, parecem não ter acontecido neste trabalho. São elas: uso de curativo compressivo no pós-operatório; imobilização do braço no pós-operatório; volume do dreno aspirativo; dias de permanência do dreno aspirativo.

Estudando pacientes que foram submetidas à cirurgia por câncer de mama, autores observaram que, dos casos com infecção, 66,6\% deles permaneceu com o dreno aspirativo por mais de 15 dias. Chamou-nos a atenção esse resultado, pois, em nosso estudo, somente quatro pacientes $(23,5 \%)$ apresentaram infecção, e o tempo de permanência do dreno não chegou a 15 dias para nenhuma das mulheres que fizeram parte da amostra. Este pode ter sido um dos motivos da baixa porcentagem de ocorrência de infecção ${ }^{(17)}$.

Verificamos que, neste estudo, a maioria das mulheres com edema do braço do lado operado eram donas-de-casa (nove - $81,8 \%$ ), iniciaram as atividades precocemente e sem moderação, foram submetidas a tratamentos complementares à cirurgia como quimioterapia e/ou radioterapia (dez - 90,9\%), apresentavam a doença em estágios de desenvolvimento II, III e IV (dez - 90,9\%). Fizeram a cirurgia do lado não dominante (oito - 72,8\%), apresentaram limitação de amplitude de movimento de braço e ombro do lado operado (nove - 81,8\%) e não faziam exercícios físicos com o braço, regularmente (dez - 90,9\%). Predominaram, ainda, as mulheres obesas, e as que fizeram mastectomia radical modificada.

Como tivemos a preocupação de fazer o acompanhamento no período de três meses de pósoperatório de cada mulher estudada, achamos oportuno, ainda, mostrar o momento em que houve aparecimento do edema, procurando relacionar com as complicações e intercorrências.

Diante dos dados podemos relatar que o edema foi precedido, coincidente ou aumentado com a ocorrência de complicações, intercorrências, e outros fatores, que foram os seguintes: ceroma; deiscência; dor contínua no local cirúrgico/braço do lado operado; infecção; limitação de amplitude de movimento de braço e ombro; aderência; realização de atividades domésticas ou outras, sem moderação; presença de secreção no local cirúrgico/local de inserção do dreno; flebite; queimadura; micose de unha; problemas com o dreno aspirativo; hematoma; sinais de inflamação no local cirúrgico; não realização de exercícios com o braço do lado operado.

Os dados registrados em nosso estudo levamnos a acreditar que a presença de complicações e intercorrências em mulheres submetidas à cirurgia por câncer de mama, podem estar contribuindo para o aparecimento do linfedema de braço. Assim, reforçamos a importância de intensificar as orientações sobre os cuidados com o braço, mesmo antes da cirurgia, para prevenir complicações futuras.

Consideramos que essas orientações devem ser feitas com explicações acessíveis à paciente, conscientizando-a do risco do edema do membro superior, e que elas têm importância primordial no programa de reabilitação da mulher submetida à cirurgia por câncer de mama.

É importante destacar o papel da enfermagem na profilaxia do linfedema, como os cuidados com o curativo cirúrgico e com o dreno aspirativo; cuidados de proteção da área a ser exposta durante a radioterapia; atividades educativas, relativas aos cuidados e realização de exercícios com o braço homolateral à cirurgia ${ }^{(4)}$. 
Os cuidados de enfermagem devem se dirigir tanto à prevenção como ao tratamento do linfedema, mas que a ênfase deve ser dada aos cuidados preventivos ${ }^{(18)}$, pois além de prejuízos físicos, o linfedema torna-se objeto de preocupação para as mulheres mastectomizadas, uma vez que, diante de sua ocorrência ele se constitui num sinal visível e representativo do câncer de mama. Ele dá visibilidade interna e externa à doença, condição importante para o enfrentamento da mesma ${ }^{(19)}$.

Todo o caminhar para a realização deste trabalho e os resultados obtidos, bem como a experiência no atendimento a mulheres submetidas à cirurgia por câncer de mama, revelaram-nos a importância e a necessidade de um acompanhamento de enfermagem a essas mulheres, principalmente no período pós-operatório.

Um melhor preparo dos profissionais de enfermagem faz-se necessário para que eles possam orientar, adequadamente, essas pacientes quanto aos cuidados com o membro superior homolateral à cirurgia, com o local cirúrgico e com o dreno aspirativo, proceder à avaliação e diagnóstico de sinais de edema, além de fornecer apoio emocional. Torna-se, também, importante a conscientização desses profissionais quanto à necessidade de aprimoramento para atender essa população.

Pensamos que essa é uma das formas de ajudar a prevenir e detectar precocemente o linfedema de braço pós-cirurgia por câncer de mama, pois acreditamos que a baixa freqüência de linfedema severo e moderado, apresentada por nossa clientela, possa estar relacionada à nossa vigilância constante nos três meses de pósoperatório, ocasião da coleta de dados.

Por outro lado, gostaríamos de ressaltar que a oportunidade de fazer o acompanhamento dessas mulheres reforçou-nos a convicção de que a enfermeira exerce um importante papel, não só na execução e orientação dos cuidados pós-operatórios, a curto e a longo prazo, mas também na articulação entre os diversos serviços destinados ao atendimento a essa clientela, especialmente aqueles que se destinam ao processo de reabilitação, como os grupos de apoio social.

Tornou-se claro para nós que novos estudos devem ser ampliados, tanto em relação ao tamanho amostral como em relação ao tempo de acompanhamento, para que se possa obter uma melhor compreensão do desenvolvimento do linfedema. Outras facetas do processo de recuperação da mulher pós tratamento cirúrgico para o câncer de mama necessitam ser estudadas, e dentre elas destacamos a adesão às atividades de reabilitação física e o papel da família na prevenção do linfedema e outras complicações.

\section{CONCLUSÕES}

Apesar de os dados deste trabalho se mostrarem pouco representativos numericamente em relação ao número amostral de sujeitos estudados, ou seja, 17 mulheres, eles nos permitiram verificar:

Complicações pós-operatórias, tais como: limitação de amplitude de movimento de braço e ombro $(64,7 \%)$; deiscência (47\%); ceroma $(41,1 \%)$; aderência $(41,1 \%)$; dor $(41,1 \%)$; infecção $(23,5 \%)$; inflamação no local de inserção do dreno aspirativo (11,8\%); celulite (5,9\%). Intercorrências como: presença de secreção no local cirúrgico ou no local de inserção do dreno (54,5\%); outros problemas com o dreno aspirativo $(27,3 \%)$; cortes, contusões, punções venosas e picadas de inseto $(27,3 \%)$; flebite $(18,2 \%)$; hematoma (18,2\%); queimadura $(9,09 \%)$; micose de unha $(9,09 \%)$. Outros fatores como: radioterapia e quimioterapia (82,3\%); não realização de exercícios com o braço do lado operado $(76,5 \%)$; estadiamento avançado da doença (III e IV - 64,7); cirurgia do lado não dominante $(64,7 \%)$; realização de cirurgias com dissecção extensa (58,8\%); obesidade $(52,9 \%)$; aumento de atividade ocupacional, sem moderação (27,3\%). Edema de mais de $1 \mathrm{~cm}$ do membro superior homolateral à cirurgia foi uma complicação que atingiu onze $(64,7 \%)$ das 17 mulheres que participaram do estudo. O linfedema severo não acometeu nenhuma delas, enquanto que o linfedema de grau moderado foi apresentado por duas mulheres (11,8\%), e as outras nove mulheres $(52,9 \%)$ tiveram linfedema de grau leve. Apesar da baixa freqüência de edema de graus moderado e severo, entre as mulheres, sujeitos de nosso estudo, sabemos que mesmo o edema de grau leve, se não tratado, adequadamente, poderá se tornar um problema sério.

Pudemos constatar, ainda, que, dentre as mulheres com edema, houve predomínio daquelas que apresentaram limitação de amplitude de movimento de braço e ombro do lado operado $(81,8 \%)$, e que não praticavam exercícios físicos, regularmente, para reabilitação $(90,9 \%)$. 
A maioria das mulheres com edema eram donasde-casa que iniciaram suas atividades precocemente e sem moderação $(81,8 \%)$, tinham sido submetidas a tratamentos com quimioterapia e/ou radioterapia (90,9\%) e apresentavam a doença em estágios II, III, IV (90,9\%). Grande parte das mulheres com edema era obesa (54,5\%), fez cirurgia com dissecção mais extensa (54,5\%) e do lado não dominante $(72,8 \%)$. Houve ainda entre as

\section{REFERÊNCIAS BIBLIOGRÁFICAS}

1. Kissin MW, Querci della Rovere G, Easton D, Westbury G. Risk of lymphoedema following treatment breast cancer. $\mathrm{Br} \mathrm{J}$ Surg 1986; 73(7):580-4.

2. Woods M, Tobin M, Mortimer P. The psychosocial morbidity of breast cancer patients with lymphoedema. Cancer Nurs 1995; 18(3):467-71.

3. Dennis B. Acquired lymphedema: a chart review of nine women's responses to intervention. Am J Occup Ther 1993; 47(10):891-8.

4. Mamede MV. Reabilitação de mastectomizadas: um novo enfoque assistencial. [tese]. Ribeirão Preto (SP): Escola de Enfermagem de Ribeirão Preto/USP; 1991.

5. Humble CA. Lymphedema: incidence, pathophysiology, management, and nursing care. Contin Educ 1995; 22(10):1503-9.

6. Goldsmith HS, Alday ES. Role of the surgeon in the rehabilitation of the breast cancer patient. Cancer 1971; 28(6):1672-5.

7. Brennan MJ, DePompolo RW, Garden FH. Focused review: postmastectomy lymphedema. Arch Phys Med Rehabil 1996; 77:74-80.

8. Sherman Jr CD, Hossfeld DK. Câncer de mama. In: Hossfeld DK, Sherman,Jr CD, organizadores. Manual de oncologia clínica. 5. ed. São Paulo (SP): UICC Fundação Oncocentro; 1991. p. 259-78.

9. Markiewicz DA, Schultz DJ, HaasJA, Harris EER, Fox KR, Glick JH, et al. The effects of sequence and type of chemotherapy and radiation therapy on cosmesis and complications after breast conservation therapy. Int J Rad Oncol Biol Phys 1996; 35(4):661-8.

10. Segerström K, Bjerle, Graffman S, Nyström A. Factors that influence the incidence of brachial oedema after treatment of breast cancer. Scand J Plast Reconstr Hand Surg 1992; 26:2237.

11. Haagensen CD. Resultados com a mastectomia radical de Halsted In: Haagensen CD. Doenças da mama. 3. ed. São Paulo (SP): Rocca; 1989. p.929-57.

12. Chilson TR, Chan FD, Lonser RR, Wu TM, Aitken DR. Seroma prevention after modified radical mastectomy. Am Surg 1992; 58(12):750-4.

13. Sasaki T, Lamari NM. Reabilitação funcional precoce pós mastectomia. HB Científica 1997 mai/jun/jul/ago; 4(2):121-7. 14. Leddy SK. Incentives and barriers to exercise in women with a history of breast cancer. Oncol Nurs Forum 1997; 24(5): 885-9. mulheres com edema, a presença de secreção no local cirúrgico ou de inserção do dreno (54,5\%) e 54,5\% tinham queixas constantes de dor no período pós-operatório. $\mathrm{Na}$ análise específica do grupo que apresentou edema foram observados os seguintes índices: ceroma $(45,4 \%)$; problemas com o dreno aspirativo $(45,4 \%)$; deiscência $(36,4 \%)$; infecção $(27,3 \%)$; aumento de atividade, sem moderação $(27,3 \%)$ e hematoma $(18,2 \%)$.

15. Lorenzatto AM, Rocha ACP, Oliveira AC, Fonseca ALA, Amim Junior J, da Silva LGP. Complicações imediatas no pós operatório da mastectomia. J Bras Ginecol 1995 out; 105(10):431-6.

16. Hoe AL, Iven D, Royle GT, Taylor I. Incidence of arm swelling following axillary clearance for breast cancer. Br J Surg 1992 mar; 79(3):261-2.

17. Aguillar OM, Santos BMO, de Andrade RHE, Barbosa ES. Infecção da ferida cirúrgica - uma complicação da mastectomia. Rev Bras Enfermagem 1995 abr/jun; 48(2):127-33.

18. Granda C. Nursing management of patients with lymphedema associated with breast cancer therapy. Cancer Nurs 1994; 17(3):229-35.

19. Almeida AM, Mamede MV, Panobianco MS, Prado MAS, Clapis MJ. Construindo o significado da doença: a experiência de mulheres com câncer de mama. Rev Latino-am Enfermagem 2001 setembro-outubro; 9(5):63-9. 